\title{
Assessment of total anomalous pulmonary venous connection by two-dimensional echocardiography
}

\author{
J F SMALLHORN, G R SUTHERLAND*, G TOMMASINI†, \\ $S$ HUNTER *, R H ANDERSON ${ }^{*}$ F J MACARTNEY
}

\begin{abstract}
From the The Hospital for Sick Children, Great Ormond Street, London, and the Freeman Hospital, Newcastle upon Tyne
\end{abstract}

SUMMARY Twenty-three patients with total anomalous pulmonary venous connection were studied by two-dimensional echocardiography. In all cases the diagnosis was made before invasive procedures, with surgical or angiocardiographic confirmation. Eleven patients had supracardiac drainage (three to the coronary sinus, two to the right atrium,) and seven had infracardiac drainage. In the majority of cases the precise pattern of drainage could be identified by combining suprasternal, praecordial, and subcostal views. In 12 cases where the suprasternal cut was used a pulmonary venous confluence could be identified, having a cross-like structure in nine, with three others appearing as a dilated channel behind and separate from the left atrium. Thus, twodimensional echocardiography reliably makes the diagnosis of total anomalous pulmonary venous connection and in the majority the precise pattern of drainage can be determined.

Total anomalous pulmonary venous drainage may mimic many cardiac and non-cardiac conditions particularly during infancy and the neonatal period. Traditionally angiocardiography has been necessary to confirm the diagnosis and outline the precise anatomy. ${ }^{1} \mathrm{M}$-mode echocardiography has provided some clues. ${ }^{2}$ but the correct diagnosis cannot always be made and determining the precise mode of drainage is usually impossible.

Two-dimensional echocardiography enables a detailed anatomical assessment of the pulmonary venous drainage. ${ }^{34}$ Theoretically, with a combination of suprasternal, parasternal, and subcostal views, it should be possible not only to make a positive diagnosis, but to trace the course of the anomalous venous connection. If such detailed information is possible then the need for more invasive procedures, particularly in the neonatal period, must be questioned.

The aims of this study were firstly, to determine the reliability of this technique in the general diagnosis of

\footnotetext{
* Present address: Freeman Hospital, High Heaton, Newcastle upon Tyne NE7 7DN.

† Present address: Divisione di Cardiochirurgia Infantile, Ospedale Civile, Massa, Italy.

¥ Present address: Cardiothoracic Institute, Fulham Road, London SW3 6HP.

Received for publication 18 August 1981
}

total anomalous pulmonary venous drainage, and secondly to see how accurately the precise site of drainage could be delineated.

\section{SUBJECTS}

During a 12 month period, 23 children with total anomalous pulmonary venous drainage were identified during routine echocardiography at The Hospital for Sick Children, Great Ormond Street, and the Freeman Hospital, Newcastle upon Tyne. In all cases the diagnosis was made before any invasive procedure. All but two patients were less than 5 months old. The children were studied with either an Advanced Technology Laboratory mechanical sector scanner using a 5 or $3.0 \mathrm{MHz}$ transducer or a Toshiba phased-array system using a $2.4 \mathrm{MHz}$ scan head.

A combination of praecordial, subcostal, and suprasternal cuts was employed to establish the presence of either normal or abnormal pulmonary venous drainage.

\section{PRAECORDIAL}

The transducer was initally placed in the parasternal long axis position to study the region of the coronary sinus and the floor of the left atrium. The normal coronary sinus runs in the atrioventricular groove and hence enlargement, extending superiorly, occurs from 
this point. By then moving the scan head to a more lateral position on the chest and rotating clockwise into a four chamber cut, the presence of some normal pulmonary veins or a venous confluence, if present, could be visualised.

\section{SUBCOSTAL}

Next the transducer was placed in the subcostal position and a four chamber cut obtained (Fig. 1). Here, as in the apical position, normal pulmonary veins or a venous confluence, if present, could be seen (Fig. 2). Visualisation of a confluence could be facilitated by movement of the transducer in an anteroposterior plane. The transducer was then rotated anticlockwise (Fig. 3) until the aorta and inferior vena cava could be seen in the long axis. The inferior vena cava could be followed to the right atrium, with the aorta continuing superiorly. If a descending vein below the diaphragm is present, its position may be variable. Therefore, visualisation depends on scan-

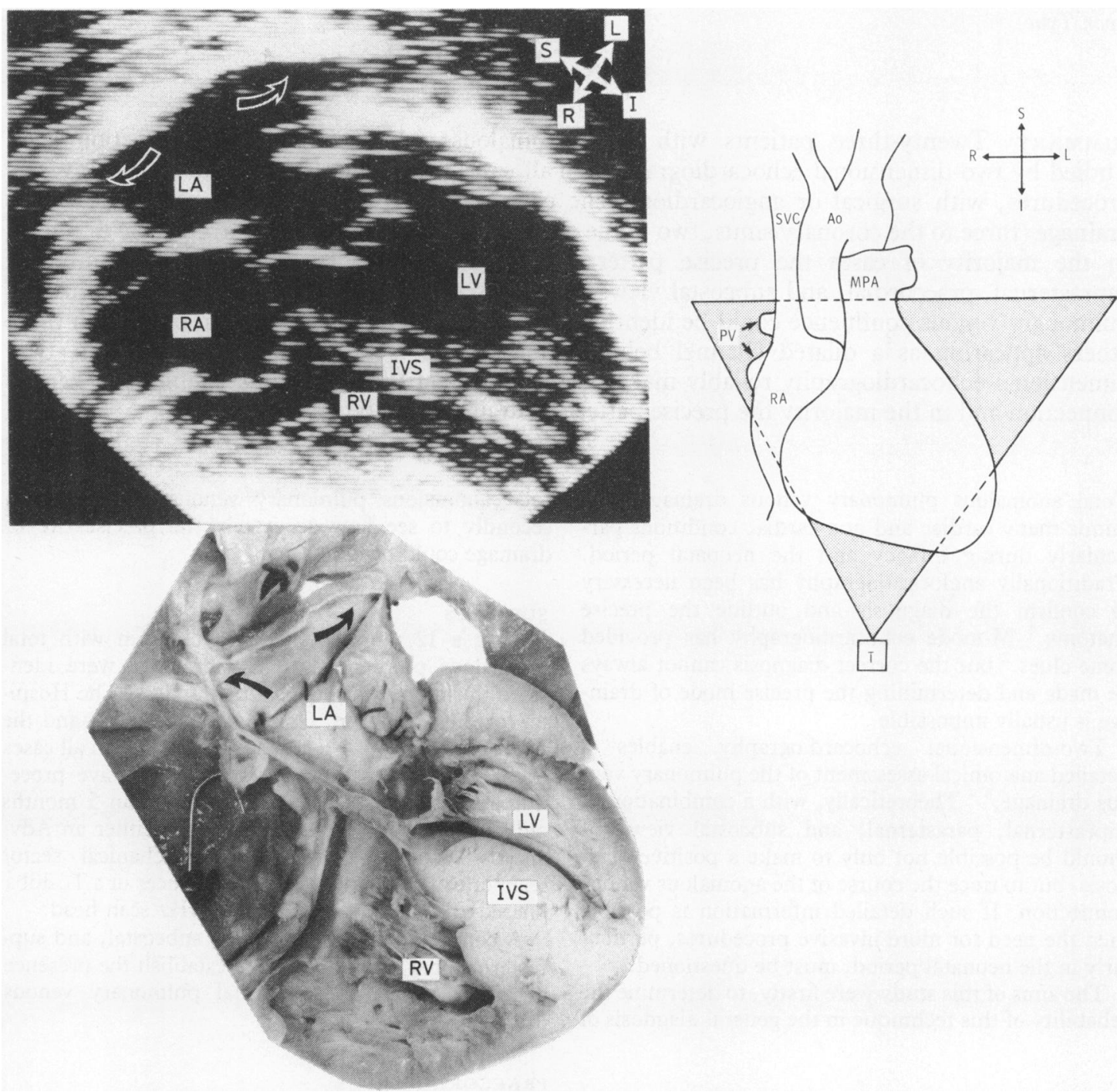

Fig. 1 The picture shows the normal pattern of pulmonary venous drainage as seen from the subcostal cut. The upper left panel shows the right upper lobe vein entering next to the interatrial septum, with the left lower lobe vein being positioned more inferiorly and to the left. The lower panel is a specimen showing the same pattern of drainage. The image on the right shows the transducer position during the examination. Ao, aorta; IVS, interventricular septum; $L A$, left atrium; $L V$, left ventricle; $M P A$, main pulmonary artery; $P V$, pulmonary veins; $R A$, right atrium; $R V$, right ventricle; SVC, superior vena cava. Arrows indicate pulmonary veins. 


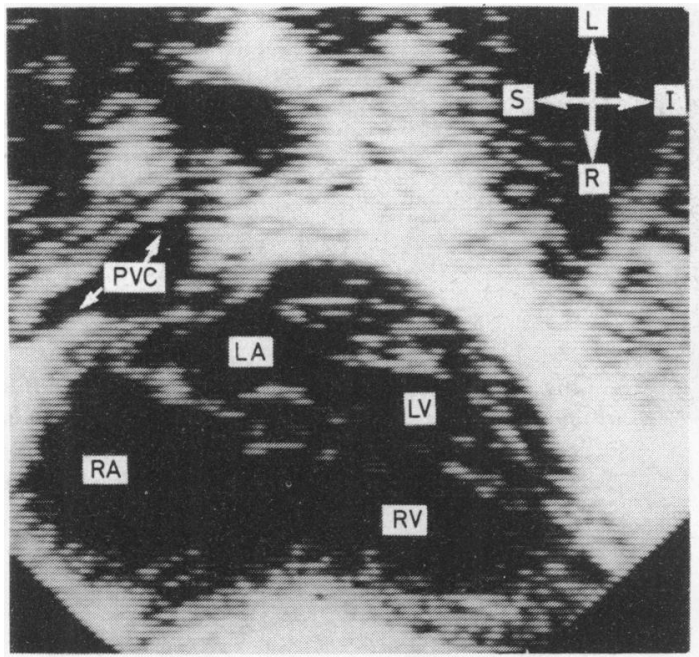

Fig. 2 A subcostal four chamber cut showing the pulmonary venous confluence behind the left atrium. The left atrium is small and has no direct contact with the confluence. PVC, pulmonary venous confluence. See Fig. 1 for remaining abbreviations.
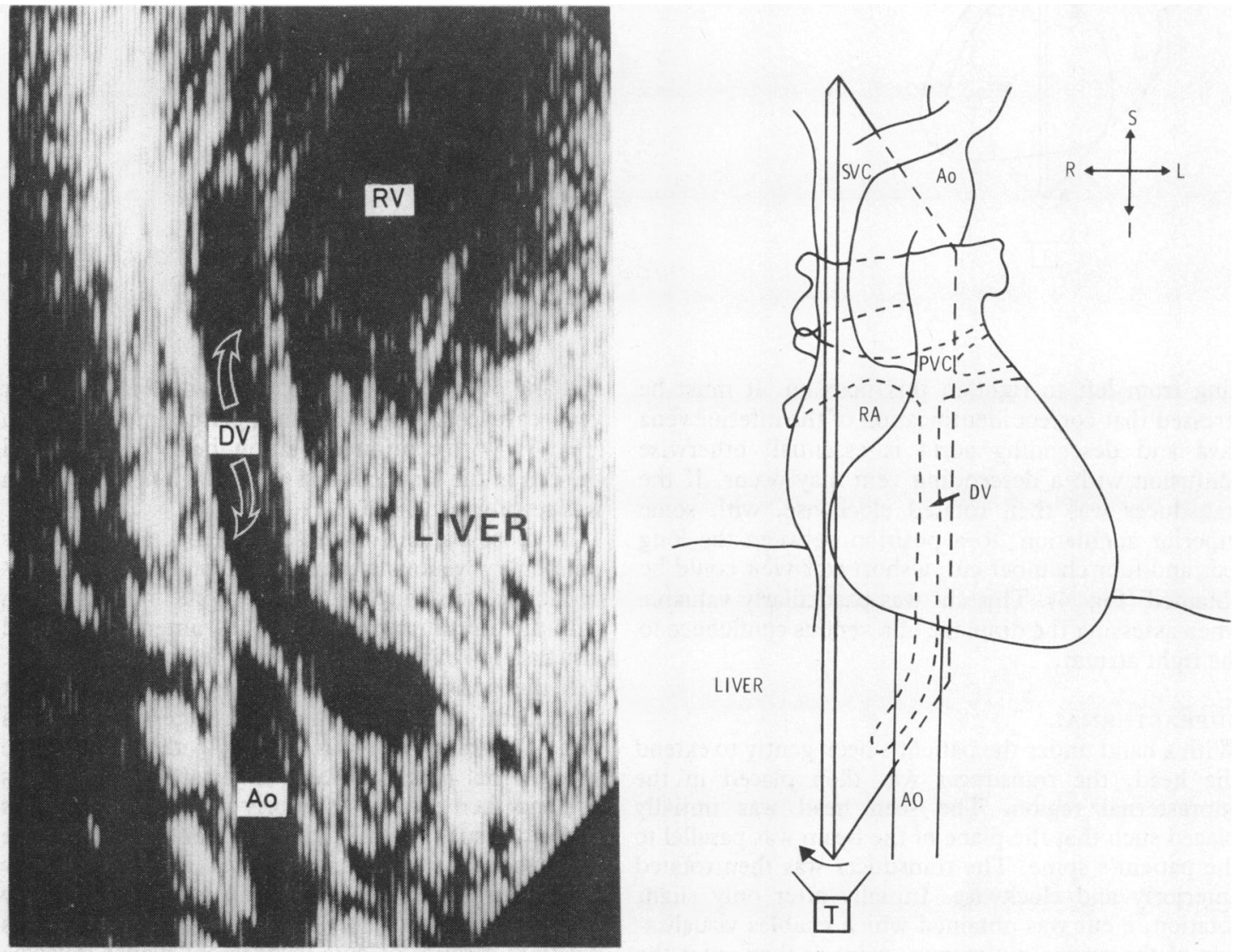

Fig. 3 Subcostal cut with the transducer angled counterclockwise visualising the aorta in a long axis. The descending vein can be seen projecting into the liver and crossing in front of the aorta. The origin of the beam is to the right of the picture. The diagram on the right shows the ultrasound plane used to obtain this view. $D V$, descending vein. See Figs 1 and 2 for remaining abbreviations. 


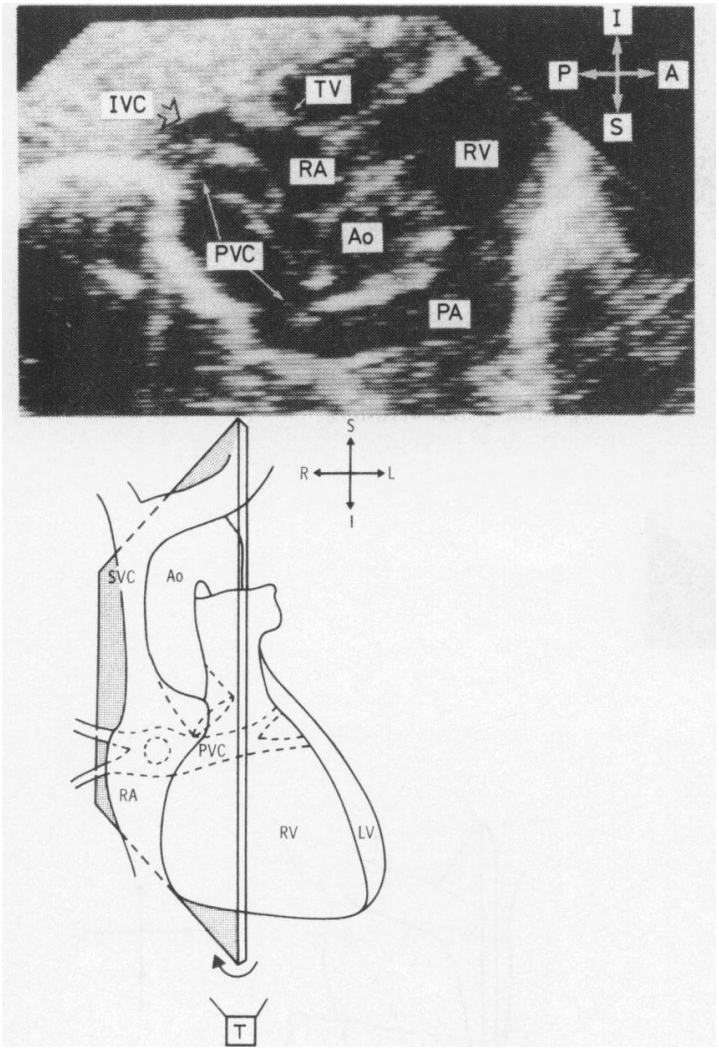

ning from left to right in this position. It must be stressed that correct identification of the inferior vena cava and descending aorta is essential, otherwise confusion with a descending vein may occur. If the transducer was then rotated clockwise, with some superior angulation, to a position between the long axis and four chamber cut, a short axis view could be obtained (Fig. 4). This cut was particularly valuable when assessing the drainage of a venous confluence to the right atrium.

\section{SUPRASTERNAL}

With a hand under the patient's neck gently to extend the head, the transducer was then placed in the suprasternal region. The scan head was initially placed such that the plane of the beam was parallel to the patient's spine. The transducer was then rotated anteriorly and clockwise. Initially after only slight rotation, a cut was obtained which enables visualisation of the normal pulmonary veins as they enter the left atrium (Fig. 5). In this position the aorta was seen in a short axis and the superior vena cava and right pulmonary artery in a long axis. The innominate vein
Fig. 4 Subcostal cut with angulation of the transducer superiorly and counterclockwise in the short axis. The venous confluence entering the right atrium can be seen. The diagram shows the position of the confluence and the ultrasound plane used during the examination. See Figs 1 and 2 for abbreviations. and any potential communication with a left superior vena cava could be seen during this initial rotation (Fig. 6). With continued rotation the aortic arch could be seen in the long axis and again the innominate vein was readily visualised.

In those patients with suspected total anomalous pulmonary venous drainage the pulmonary venous confluence was sought in this cut. The initial position with the transducer rotated slightly anteriorly allowed visualisation of the confluence in the majority of cases. But the position of a confluence may vary, so not infrequently minor modifications were necessary. In some the transducer was moved to the right of the suprasternal notch and angled towards the patient's left and then rotated anteriorly and clockwise. This enabled visualisation of the confluence and also the main and right pulmonary arteries (Fig. 7). To allow reliable visualisation of the venous confluence and any potential ascending or descending vein from this position, a series of cuts in different planes must be used, incorporating different transducer positions.

In 11 patients peripheral venous contrast injections were performed during the examination and the pat- 

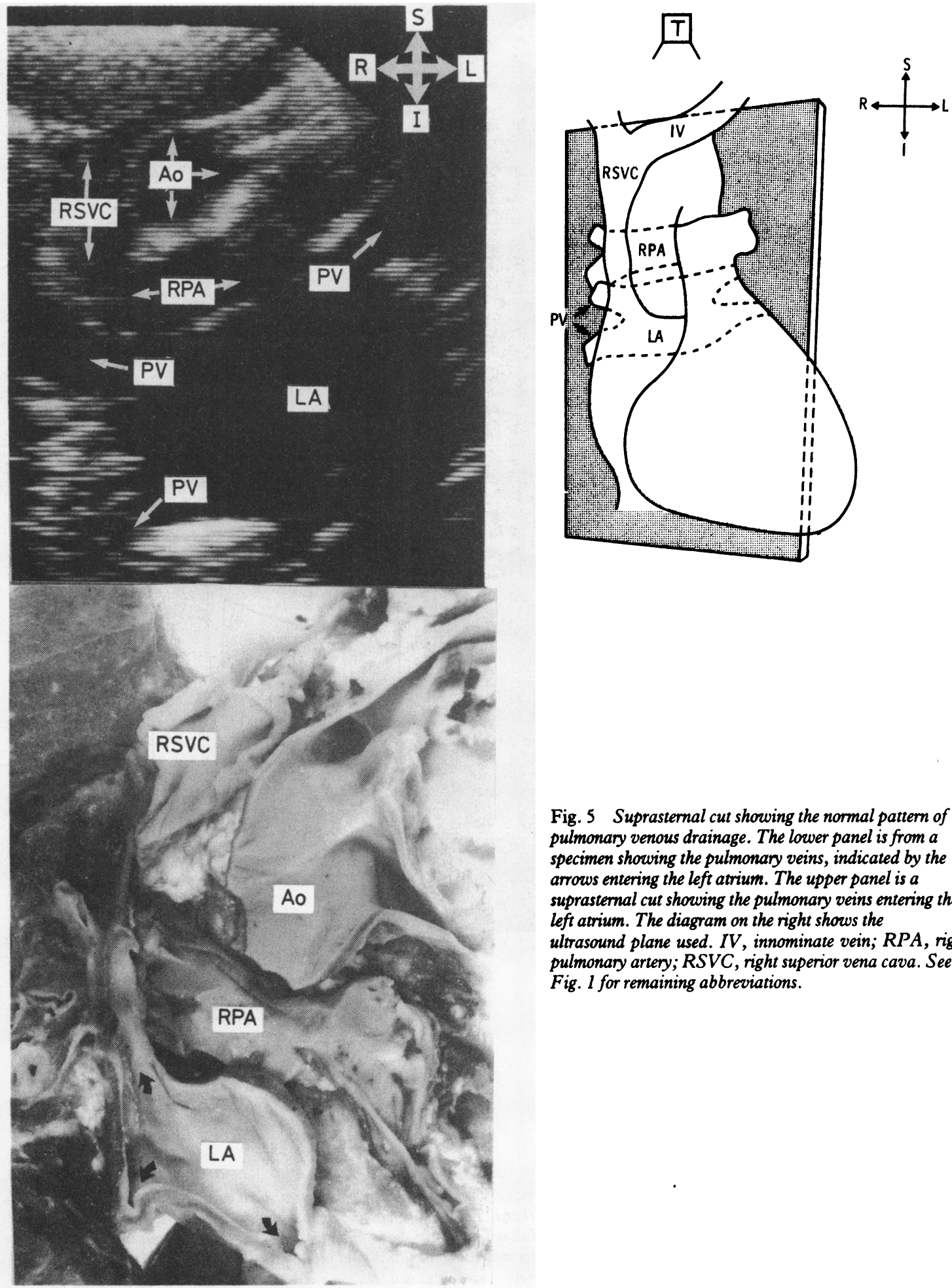

Fig. 5 Suprasternal cut showing the normal pattern of pulmonary venous drainage. The lower panel is from a specimen showing the pulmonary veins, indicated by the arrows entering the left atrium. The upper panel is a suprasternal cut showing the pulmonary veins entering the left atrium. The diagram on the right shows the ultrasound plane used. IV, innominate vein; RPA, right pulmonary artery; RSVC, right superior vena cava. See Fig. 1 for remaining abbreviations. 


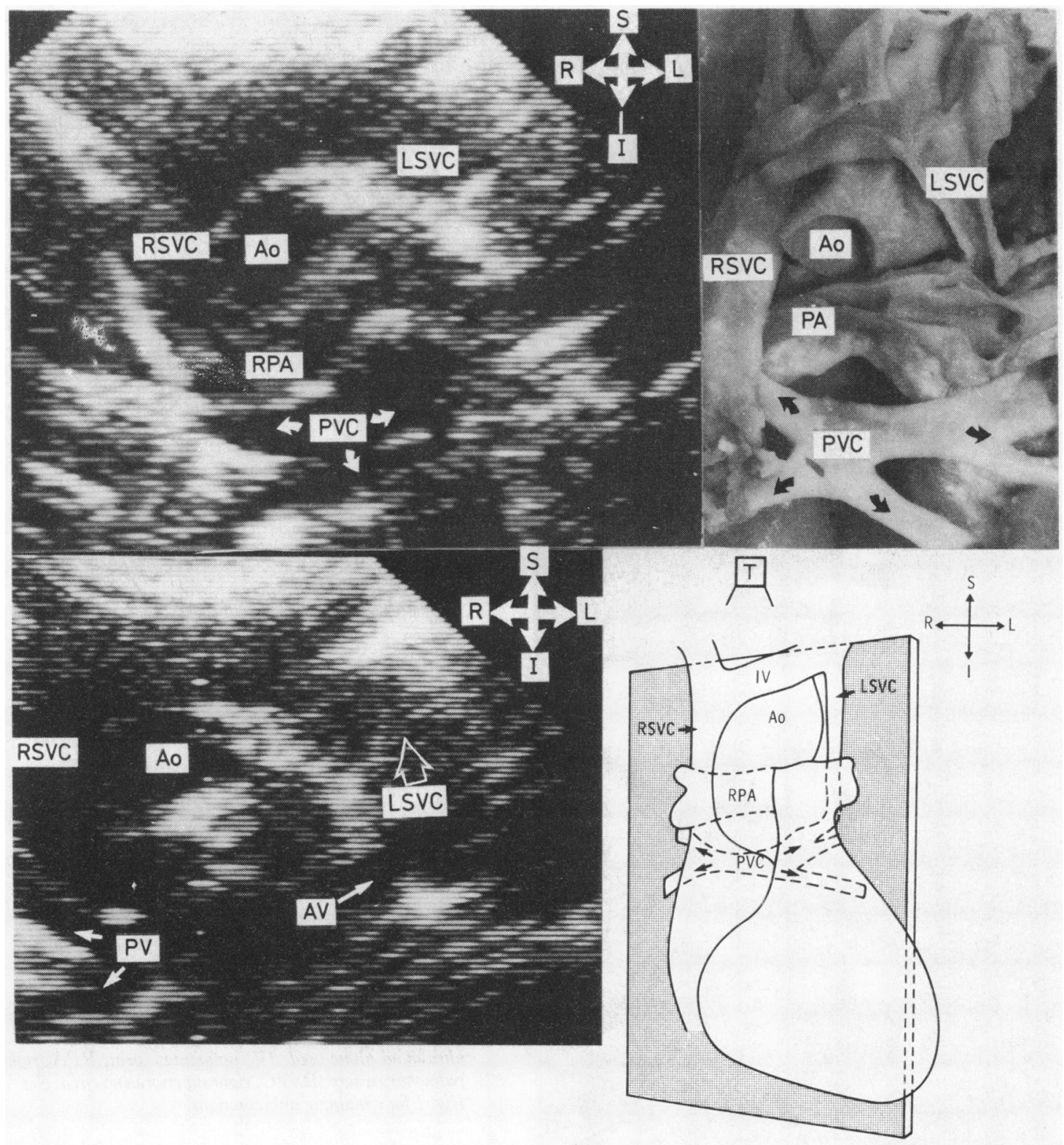

Fig. 6 Suprasternal cut in a case with total anomalous pulmonary venous drainage via a left vertical vein. The upper left panel shows the pulmonary venous confluence with its "cross-like" shape. The specimen on the right shows the left superior vena cava (left vertical vein) and the venous confluence. The left lower panel shows the site of the communication between the venous confluence and the left superior vena cava. The lower right image is a diagram indicating the ultrasound plane used. AV, ascending vein; LSVC, left superior vena cava. See Figs 1, 2, 5, and 6 for abbreviations. 

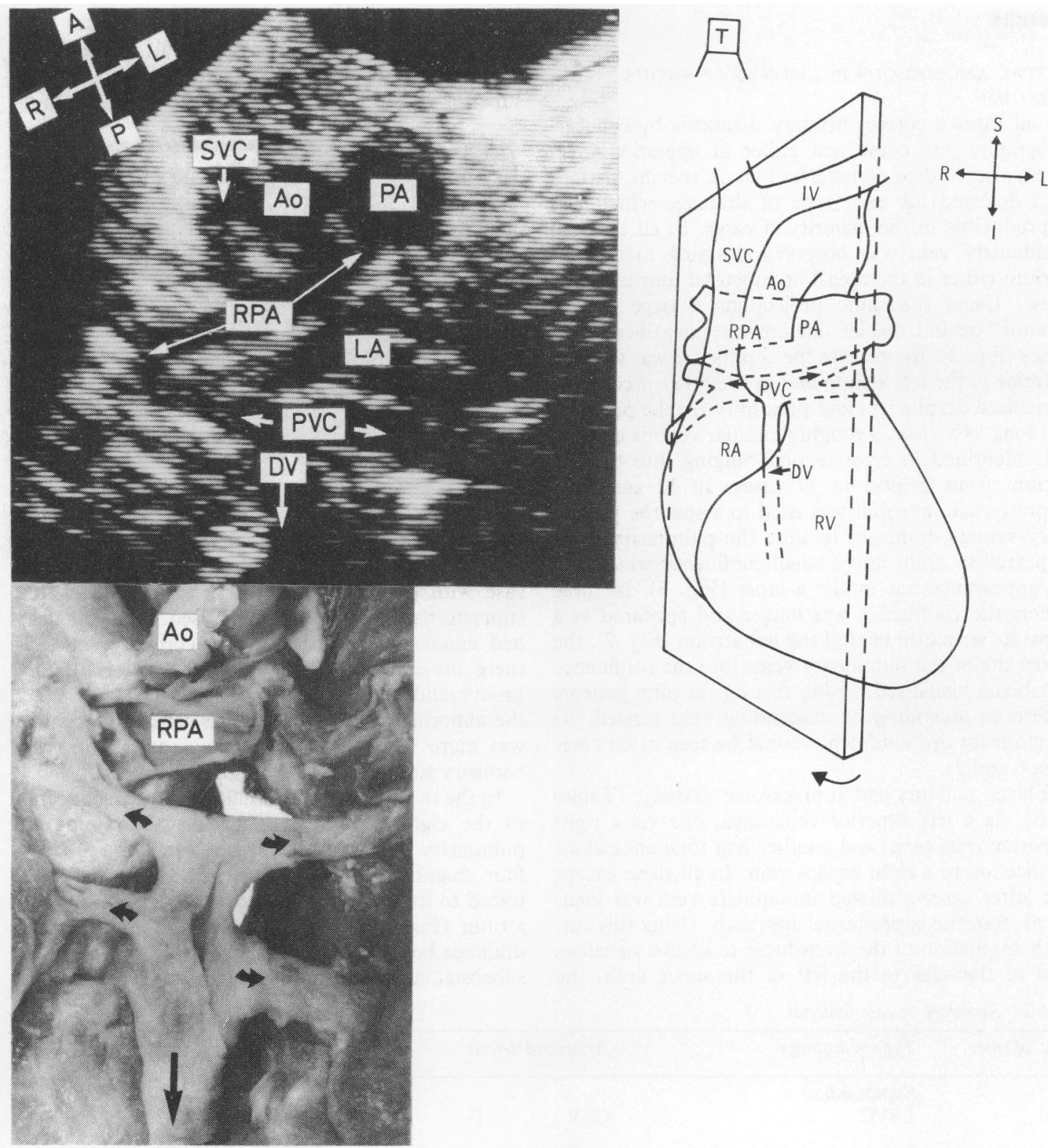

Fig. 7 Suprasternal cut in a case with total anomalous pulmonary venous drainage via a descending vein. The upper picture shows the pulmonary venous confluence seen running behind and separate from the left atrium. Note the descending vein. The lower panel is a specimen showing the venous confluence and the descending vein indicated by the large arrow. The diagram on the right shows the ultrasound plane used during the examination. See Figs 1, 2, and 5 for abbreviations.

tern of flow noted. In all patients the relative size of the ventricular and atrial chambers was observed, as were any other associated anomalies. All patients had angiocardiographic and or surgical demonstration of the exact mode of pulmonary venous connection.

When there is pulmonary venous confluence behind the left atrium in these patients, or pulmonary venous return is to the coronary sinus, two chambers are seen in place of the normally undivided left atrium. Since a similar situation exists in cor triatriatum, three patients with this condition are added for comparison. 


\section{Results}

TOTAL ANOMALOUS PULMONARY VENOUS RETURN

In all cases a correct primary diagnosis by echocardiography was confirmed either at operation, necropsy, or cardiac catheterisation. A specific pattern was detected for each type of drainage which was reproducible in the majority of cases. In all cases no pulmonary vein was observed draining to the left atrium either in the apical or subcostal four chamber view. Using the same projections a large venous channel behind the left atrium was identified in 21 cases (Fig. 2). In one case the confluence was situated inferior to the left atrium and in the other it could be visualised despite its close proximity. In the parasternal long axis view, a roughly circular venous channel was identified in cross-section bulging into the left atrium from behind in 17 cases. In 12 cases the suprasternal approach was used to assess the pulmonary venous drainage. In nine the pulmonary veins appeared to drain into a small confluence which had an appearance not unlike a cross (Fig. 6). In three others the confluence was longer and appeared as a separate structure behind the left atrium (Fig. 7), the entry site of the pulmonary veins into the confluence not being visualised. Using this cut in nine patients where an ascending or descending vein existed, its origin from this confluence could be seen in six cases (Fig 6 and 7).

Eleven patients had supracardiac drainage (Table) (nine via a left superior vena cava, one via a right superior vena cava) and another had total anomalous connection to a right azygos vein. In all these except the latter case, a dilated innominate vein was identified from the suprasternal approach. Using this cut, with angulation of the transducer to secure visualisation of the area to the left of the aortic arch, the persistent left superior vena cava (or vertical vein) could be seen in eight patients (Fig. 6). In one patient with supracardiac drainage the entry site to the right superior vena cava could be visualised (Fig. 8). In the

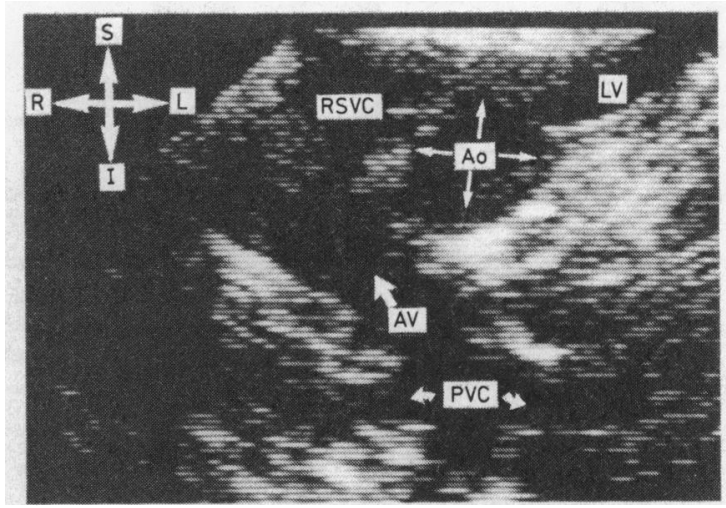

Fig. 8 Suprasternal cut showing an ascending vein to the right superior vena cava. See Figs 1, 2, 5, and 6 for abbreviations.

case with drainage to the right azygos system the suprasternal approach was not used. Three patients had anomalous drainage to the coronary sinus. In these the channel originated at the atrioventricular groove and swept anterosuperiorly (Fig. 9). When the abnormal connection was elsewhere the channel was more superior, and in many cases the normal coronary sinus could be separately identified.

In the two patients with total anomalous connection to the right atrium, the confluence draining the pulmonary veins could be clearly seen in the subcostal four chamber and parasternal short axis views and traced to its entry into the posterior wall of the right atrium (Fig. 4). Seven cases had total anomalous drainage below the diaphragm (Table). In these the subcostal cut with the transducer angled to visualise

Table Summary of case material

\begin{tabular}{lllll}
\hline No. of cases & Type of drainage & Associated defects & Ventriculoarterial connections \\
\hline 9 & Supracardiac & CAV & $(1)$ & Condordant (9) \\
1 & LSVC & UVH & $(1)$ & DORV \\
1 & RSVC & Inlet VSD & $(1)$ & DOMC \\
7 & Azygos & CAV & $(1)$ & Concordant (7) \\
& Infracardiac & & & Concordant (3) \\
3 & Intracardiac & & & Concordant (2)
\end{tabular}

Abbreviations:

CAV, complete atrioventricular septal defect; C. sinus, coronary sinus; DOMC, double outlet from main chamber; DORV, double outlet right ventricle; LSVC, left superior vena cava; RSVC, right superior vena cava; Rt. atrium, right atrium; UVH, univentricular heart. 


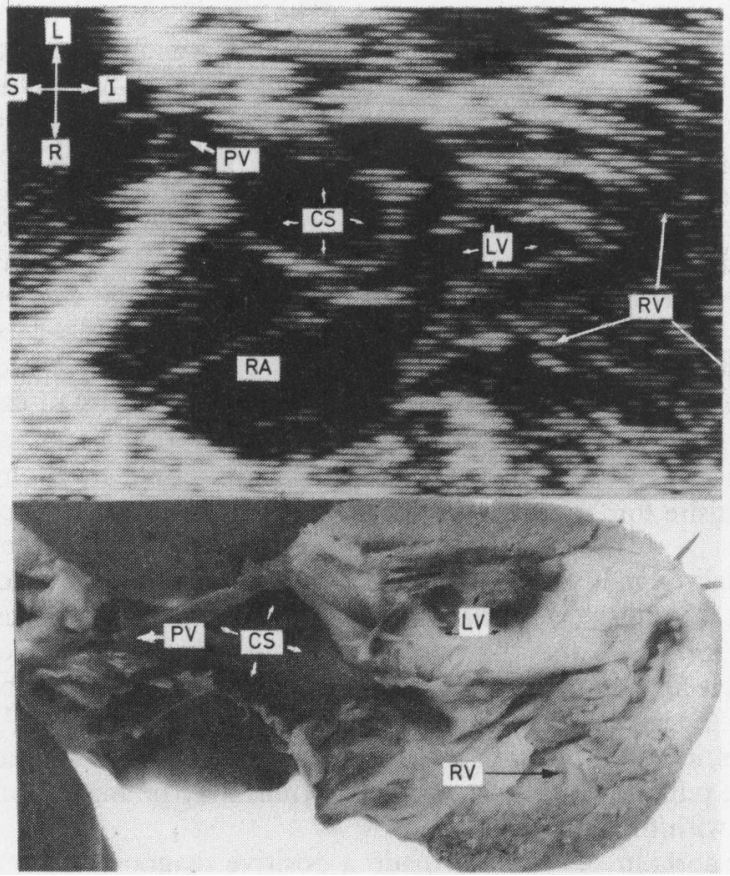

Fig. 9 These pictures show total anomalous pulmonary venous connection to the coronary sinus. The upper panel is a subcostal cut showing the dilated coronary sinus originating at the atrioventricular groove and extending superiorly. The lower panel is from a specimen with total anomalous pulmonary venous drainage to the coronary sinus. CS, coronary sinus. See Fig. I for remaining abbreviations.

the inferior vena cava, descending aorta, and right atrium, enabled five descending veins to be identified (Fig. 3). Such vessels when observed appeared to cross the inferior vena cava and aorta to protrude like a finger towards the liver. In all cases with intact septa, indirect evidence was available in the form of a small left atrium and a dilated right atrium and ventricle (Fig. 2). Contrast injections using a peripheral vein showed pronounced right-to-left shunting at atrial level. The contrast opacified the left side far more intensely than the right.

Two cases had mixed pulmonary venous drainage, both having the left sided veins draining to a vertical vein and the right to a coronary sinus. The dilated vertical veins were identified in both cases, and in one a dilated coronary sinus with pulmonary veins entering it was noted at the time of examination.

\section{COR TRIATRIATUM SINISTER}

In these three patients, the abnormal features allowed easy differentiation from those with total anomalous pulmonary venous drainage. The suprasternal view disclosed a normal drainage pattern of the pulmonary veins. In one case a persistent left superior vena cava from the superior chamber was visualised. A dense echo dividing the left atrium into a superior and inferior chamber was seen in the apical and subcostal four chamber views, with normal pulmonary venous drainage into the superior chamber being identified in all cases.

\section{ASSOCIATED ABNORMALITIES}

The atrioventricular connection was normal in all patients except one with a double inlet univentricular heart and a common atrioventricular valve. The ventriculoarterial connections were normal in 21 patients, with one other having a double outlet right ventricle and another a double outlet from the main chamber (Table). One patient had an inlet muscular ventricular septal defect and two complete atrioventricular defects.

\section{Discussion}

Total anomalous pulmonary venous drainage may be difficult to diagnose clinically, particularly in the neonatal period where other cyanotic forms of congenital heart disease may mimic its symptomatology. Probably the biggest area of confusion arises in those infants with persistent fetal circulation. Not uncommonly cardiac catheterisation was necessary in the past to differentiate these conditions.

M-mode echocardiography has provided some clues in assessing children with suspected total anomalous pulmonary venous connection. ${ }^{2}$ In most cases the right ventricle and pulmonary artery are large while the left atrium is small. Occasionally a chamber behind the left atrium can be seen and by using the suprasternal approach, a dilated innominate vein may be identified, if present. In many cases, however, confusion particularly with persistent fetal circulation 
has remained a constant problem. In the majority the precise pattern of drainage has been impossible.

Normal pulmonary venous drainage can be accurately assessed by two-dimensional echocardiography, particularly using systems with good lateral resolution. ${ }^{34}$ When faced with the prospect of identifying normal or abnormal venous drainage the precise pattern of entry must be identified. In those with a confluence behind the left atrium, a clear line of demarcation between the atrium and venous channel must be seen for a firm diagnosis to be made. From the suprasternal approach, particularly in the neonate and infant, a venous confluence can only be seen confidently with a $5 \mathrm{MHz}$ system. In our view in the neonatal period the latter system is a prerequisite for investigation.

Normally two pulmonary veins can be visualised in the subcostal view as they enter the left atrium (Fig. 1). The right upper lobe vein is seen in close proximity to the interatrial septum with the left lower lobe vein being situated more inferiorly and to the left. Likewise the apical four chamber cut allows identification of the right upper and left lower lobe pulmonary veins. From the suprasternal approach, with the transducer angled to allow visualisation of the aorta in a short axis and pulmonary artery and superior vena cava in a long axis, the right and left upper and lower pulmonary veins can be identified as they enter the left atrium (Fig. 5).

Morphologically, total anomalous pulmonary venous connection has four basic patterns of drainage, that is infradiaphragmatic, to the superior vena cava or vertical vein, right atrium, and coronary sinus. Certain variations exist and likewise combinations, but in general they follow the above patterns. ${ }^{56}$ In all varieties except mixed drainage and some cases of total connection to the right atrium, there is a common pulmonary vein into which the individual pulmonary veins drain. This usually is in close proximity to the left atrium but occasionally it may be far away, as was seen in one of our cases.

In the supracardiac type the drainage occurs via a vertical vein which ascends and joins the innominate vein (Fig. 6). Occasionally the confluence may connect directly with a right superior vena cava near its junction with the right atrium or an azygos system. When the drainage is infracardiac, the vein leaving the venous confluence descends and joins either the portal circulation or inferior vena cava (Fig. 3). Drainage to the coronary sinus results in gross dilatation of that structure which protrudes into the back of the left atrium (Fig. 9).

There appears to be no confusion between the diagnosis of cor triatriatum and total anomalous pulmonary venous drainage as they both have different patterns, with the former having normally positioned pulmonary veins. The diagnosis and assessment of total anomalous pulmonary venous drainage by twodimensional echocardiography, is not unlike piecing together a puzzle. Sahn and colleagues ${ }^{7}$ showed that certain patterns were evident, but did not use the suprasternal and subcostal approaches to trace the ascending or descending vein. In our experience, the features common to all types were the absence of normal pulmonary venous drainage as viewed from the subcostal and apical four chamber projection. Instead, a common pulmonary vein could be seen behind the left atrium in the majority of cases which immediately indicated the presence of anomalous pulmonary venous drainage (Fig. 2). From the suprasternal approach the entry pattern of the pulmonary veins forming a cross as they entered the common venous confluence was typical, and in all cases indicated that the child had abnormal drainage (Fig. 8). In others from the suprasternal cut the confluence appeared to be longer and could be seen behind the left atrium as a separate channel (Fig. 7). In this position unless the transducer is rotated slightly anteriorly the left atrium may be missed in the same cut.

Having made a positive diagnosis from these features the exact mode of connection could usually be determined. When a dilated innominate vein and left superior vena cava were visualised, this represented the supracardiac variety (Fig. 6 and 8 ). In some, the connection to the common ascending or descending vein could be seen from the suprasternal view as it left the pulmonary venous confluence (Fig. 6 and 7). In the subcostal cut it is important first to identify the descending aorta and inferior vena cava at its junction with the right atrium, so that confusion between these structures and the descending vein does not occur. Serial scans across the abdomen from left to right are necessary to pick up the abnormal vessel, but even using this technique the descending veins may be missed. They can almost always be followed as far as the diaphragm and the inability to observe them below it may possibly be the result of compression of the vessel by the liver.

If neither an ascending nor descending vein is visualised, then the possibility of drainage to either the coronary sinus or right atrium exists. The coronary sinus has a characteristic pattern, as it anatomically lies in the atrioventricular groove. Hence when it dilates because of increased flow it is always attached at that point and then bulges anterosuperiorly into the left atrium (Fig. 9). This is unlike many of the other types where the common pulmonary vein indents the left atrium in a more superior position. When the drainage was to the right atrium the channel could be seen behind the left atrium and followed towards the site of entry (Fig. 4). 
Surgically it is important to know the type of drainage and presence of associated defects, which may influence the mode of management. When the diagnosis is made by two-dimensional echocardiography, drainage to the coronary sinus or right atrium can be confirmed reliably and so can that to the superior vena cava. If the descending vein is not seen during the examination, this does not exclude total anomalous connection below the diaphragm caused by false negatives. But in the presence of the common features already described and the lack of drainage to the right atrium, coronary sinus, or supracardiac region it must logically drain below the diaphragm. It can also be argued that, providing a venous confluence has been identified, recognition of the descending vein is not of vital importance, since the surgeon will readily see this structure during the surgical intervention. One measure of our confidence in the technique is that a 2-month-old patient, with total connection to a vertical vein, was submitted to successful surgical repair on the basis of the echocardiographic findings alone, without prior cardiac catheterisation.

Certain varieties of total anomalous pulmonary venous return were not seen during the period of this study, in particular drainage to the right atrium by individual veins. It appears that mixed drainage may present difficulties, but then in our experience the same problem not infrequently occurs with angiocardiography. Peripheral contrast studies provide a high level of suspicion, particularly in the presence of a small left atrium and large right ventricle, but by itself difficulties still arise between total anomalous pulmonary venous connection and persistent fetal circulation.

When basing a surgical decision upon non-invasive techniques, the risks of failing to obtain a diagnosis which is absolute in every detail must be weighed up against the risks of invasive procedures. With reference to total anomalous pulmonary venous connection, non-invasive studies enable a correct diagnosis with some potential deficiencies in the mixed types and known problems with visualising the descending channel beneath the diaphragm. If the surgeon is willing to accept these deficiencies then the noninvasive approach should provide an adequate assessment.

Thus with two-dimensional echocardiography using a high frequency system, the diagnosis can readily be made and in the majority of cases the exact mode of drainage identified. This should in the future enable intervention without prior invasive studies being necessary.

JFS is a British Heart Foundation Junior Research Fellow. RHA and FJM are supported by the British Heart Foundation and, respectively, the Joseph Levy and Vandervell Foundations.

\section{References}

1 Edwards JE, Carey LS, Neufeld HN, Lester RG. Congenital heart disease: correlation of pathologic anatomy and angiocardiography. Philadelphia \& London: WB Saunders, 1965: 880-90.

2 Meyer RA. Total anomalous pulmonary venous return. In: Meyer RA, ed. Pediatric echocardiography. Philadelphia: Lea \& Febiger, 1977: 113-25.

3 Bansal RC, Tajik AJ, Seward JB, Offord KP. Feasibility of detailed two-dimensional echocardiographic examination in adults. Prospective study of 200 patients. Mayo Clin Proc 1980; 55: 291-308.

4 Tajik AJ, Seward JB, Hagler DJ, Mair DD, Lie JT. Two-dimensional real-time ultrasonic imaging of the heart and great vessels. Technique, image orientation, structure identification, and validation. Mayo Clin Proc 1978; 53: 271-303.

5 Bonham Carter RE, Capriles M, Noe Y. Total anomalous pulmonary venous drainage. A clinical and anatomical study of 75 children. Br Heart $\mathcal{f} 1969$; 31: 45-51.

6 Hastreiter AR, Paul MH, Molthan ME, Miller RA. Total anomalous pulmonary venous connection with severe pulmonary venous obstruction. A clinical entity. Circulation 1962; 25: 916-28.

7 Sahn DJ, Allen HD, Lange LW, Goldberg SJ. Crosssectional echocardiographic diagnosis of the sites of total anomalous pulmonary venous drainage. Circulation 1979; 60: $1317-25$.

Request for reprints to Professor F J Macartney, The Hospital for Sick Children, Great Ormond Street, London WCIN 3JH. 